\title{
MULTIPLE CLASSIFICATION ALGORITHMS UNIMODAL AND MULTIMODAL TARGET RECOGNITION SYSTEMS
}

\author{
Veaceslav Perju*, ORCID ID: 0000-0002-7755-4277 \\ Agency for Military Science and Memory, 47 Tighina Str., Chisinau, Republic of Moldova \\ *Corresponding author: Veaceslav Perju, vlperju@yahoo.com
}

Received: 06. 11. 2021

Accepted: 08. 12. 2021

\begin{abstract}
Target recognition is of great importance in military and civil applications - object detection, security and surveillance, access and border control, etc. In the article the general structure and main components of a target recognition system are presented. The characteristics such as availability, distinctiveness, robustness, and accessibility are described, which influence the reliability of a TRS. The graph presentations and mathematical descriptions of a unimodal and multimodal TRS are given. The mathematical models for a probability of correct target recognition in these systems are presented. To increase the reliability of TRS, a new approach was proposed - to use a set of classification algorithms in the systems. This approach permits the development of new kinds of systems - Multiple Classification Algorithms Unimodal and Multimodal Systems (MAUMS and MAMMS). The graph presentations, mathematical descriptions of the MAUMS and MAMMS are described. The evaluation of the correct target recognition was made for different systems. The conditions of systems' effectiveness were established. The modality of the algorithm's recognition probability maximal value determination for an established threshold level of the system's recognition probability was proposed, which will describe the requirements for the quality and, respectively, the costs of the recognition algorithms. The proposed theory permits the system's design depending on a predetermined recognition probability.
\end{abstract}

Keywords: target, recognition, system, unimodal, multimodal, algorithm, probability.

\section{Introduction}

Target recognition is of great importance in military and civil applications - object detection [1], image classification [2], security and surveillance [3], access control [4], border control[5], medicine[6], etc. Actual target recognition systems can be divided into two main groups - unimodal and multimodal systems[7]. The unimodal systems are based usually on one processing module and one recognition algorithm [8]. The multimodal systems consist of a set of sensors and processing modules, every of which is realizes one target recognition algorithm [9].

In many unimodal and multimodal target recognition systems, the operations of the data acquisition and processing are realized as separated tasks. As a result, the accuracy and robustness of the target recognition may be insufficient, especially if the data are found to 
be incomplete or ambiguous during processing and there are no available supplementary data to mitigate those deficiencies [10]. Active multimodal sensor systems have been elaborated to overcome this problem. For example, a multisensory system for moving object detection and tracking was proposed [11] in which were used Radar, LIDAR, and vision sensors.

The system could actively control all the sensors to collect or supplement target information and effectively detect and track target movement, and obtained good performance results in actual driving conditions. The multimodal sensing system was elaborated [12], using a PTZ camera and a laser Doppler vibrometer, which can improve the performance and efficiency in automatic remote applications. This class of systems is based on the combination of multimodal hardware data collection. Subsequent data processing permits improvement of the accuracy of recognition and tracking systems, to expand the application range, and increase the system's flexibility. In other surveillance systems, the hardware data collection and software data processing are two completely separate and independent parts [13]. In these systems, the software data processing algorithms are used to improve the targets' detection and tracking rate, and the hardware sensor is used only for data collection and does not consider the interaction with the sensor to supplement the data and eliminate ambiguous judgments.

In the article [14] an active multimodal sensor system for target recognition and tracking was proposed. Different from the passive multi-modal sensor system, the active multi-modal system can adjust sensors' attitudes to get supplementary information during data processing. This system consists of a visible sensor, an infrared sensor, and a hyperspectral sensor, working together to collect supplementary data from the target to eliminate ambiguous recognition.

Unfortunately, in many applications, the described systems do not ensure the necessary level of identification probability, accuracy, and robustness of the target recognition may be insufficient.

In this article new classes of the systems are described - multiple classification algorithm unimodal and multimodal systems and results of a comparative analysis of the proposed and existing systems regarding their recognition probability are presented. In section 2 the general structure and main components of a target recognition system are presented. The characteristics such as availability, distinctiveness, robustness, and accessibility are described, which influence the reliability of a TRS. In sections 3 and 4 the graph presentations and mathematical descriptions of a unimodal and multimodal TRS are given. The mathematical models for a probability of correct target recognition in these systems are presented. It was determined that the unimodal systems are characterized by a disadvantage - a vulnerability of the sensor to bad or noisy data as a result of the imperfect acquisition of captured features. In section 5 the new classes of the systems are described multiple classification algorithm unimodal and multimodal systems (MAUMS and MAMMS). The graph presentations, mathematical descriptions of the MAUMS and MAMMS are described. In section 6 the evaluation of the correct target recognition was made for different systems: the unimodal system (UMS); multimodal systems with one algorithm and 2 and 3 sensors (MMS-1A2S and MMS-1A3S); multi-algorithm unimodal systems with 2 and 3 algorithms and one sensor (MAUMS-2A1S and MAUMS-3A1S), multi-algorithm multimodal systems with 2 algorithms and 2 sensors (MAMMS-2A2S), with 3 algorithms and 2 sensors (MAMMS-3A2S) and with 3 algorithms and 3 sensors (MAMMS-3A3S). The conditions of 
systems' effectiveness were established. The modality of the algorithm's recognition probability $\mathrm{P}_{\mathrm{AM}}$ maximal value determination for an established threshold level of the system's recognition probability $P_{S T}$ was proposed. The values of $p_{A M}$ will describe the requirements for the quality and, respectively, the costs of the recognition algorithms.

\section{General structure of a target recognition system}

A target recognition system (TRS) acquires the data of an object, extracts a feature set from the data, compares this feature set against the feature sets stored in the database, and provides the results of the comparison [15]. A TRS consists of the next main components (Figure 1) [16]: sensor unit, which acquires the data of an object by scanning; quality assessment and feature extraction unit, which is used for further processing of the input data; local decision-making unit, in which the extracted templates are then matched against the stored templates and a matching score is given; output decision-making unit, in which the data from one or more local decision-making modules are processed and the final identity of the target is made; system database unit; the basic software, intended for general management of the processes in the system; the algorithmic software, used to realize the target recognition tasks.

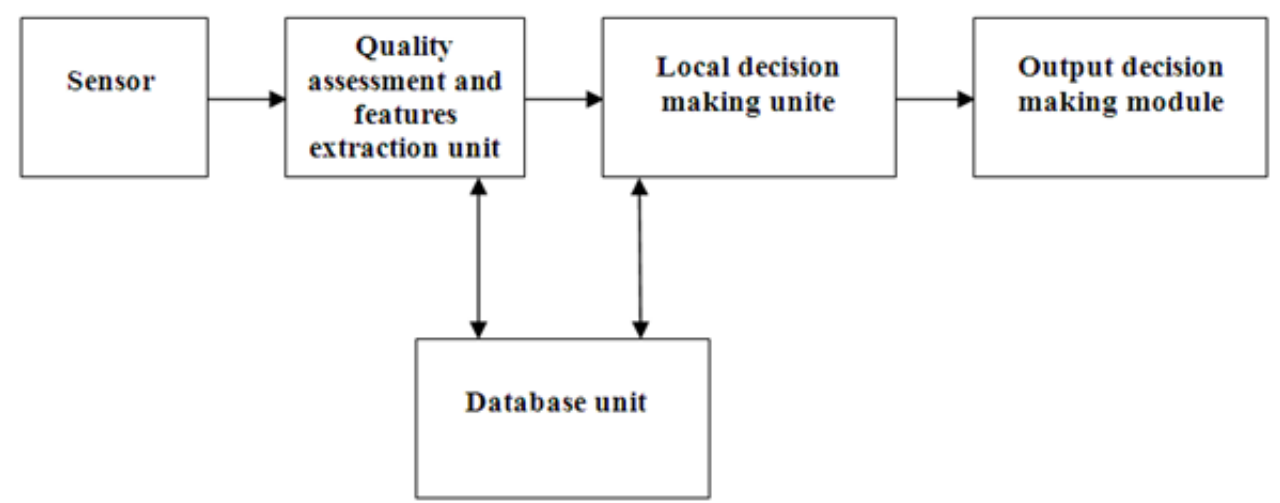

Figure 1. The structure of the target recognition system.

A target recognition system can consist of one or more sensors and processing modules, which include the units described previously. The reliability of a TRS depends on the following characteristics [17]. Availability - indicates that a target should have distinct characteristics. Distinctiveness - this asserts that two targets should have adequately different characteristics. Robustness - it declares that characteristics should be constant over some time concerning matching characteristics. Accessible - it asserts that the features can be measured using a quantitative method, and can also be easy to image with electronic sensors.

Unfortunately, not all the described requirements can always be achieved at the different stages of the target image acquisition and processing, which is reflected in the probability level of the target's recognition.

\section{Model of the unimodal target recognition system}

The unimodal target recognition system consists of one sensor for target image acquisition, one processing module, in which is realized one recognition algorithm, and one output decision-making unit [18]. The processing module includes a quality assessment and feature extraction unit, local decision-making unit, and database unit. The unimodal system can be presented in the form of a graph shown in Figure 2. 


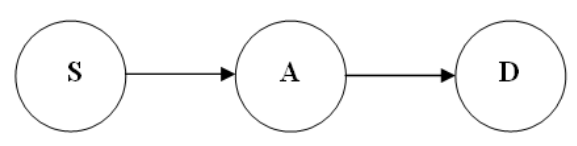

Figure 2. The graph presentation of the unimodal system: $\mathrm{S}$ - sensor, A - algorithm,

$\mathrm{D}$ - output decision-making module.

Let $T(x, y)$ be the function that describes the input target. Following algorithm $A$ of target recognition, at the first stage will be extracted the features $F_{A}=\left\{f_{A i}\right\}, i=1 \div 1$ from the function $T(x, y)$. At the second stage will be determined the matrix $D_{A}=\min \left\{W\left[F_{A}, F_{A j}\right]\right\}$, were $F_{A j}=\left\{f_{A j}\right\}$ - features of the reference targets, $j=1 \div$ J. At the next stage, the input target will be identified. The probability of correct recognition of the target $T(x, y)$ in the system will be:

$$
\mathrm{P}_{\text {UMS }}=\mathrm{p}_{\mathrm{A}}
$$

where $\mathrm{p}_{\mathrm{A}}$ is the probability of correct recognition of the target based on algorithm $A$.

In article [19] the recognition system, based on the Image Moments Features algorithm, principal component analyses algorithm, and correlation algorithm. The software application captures the target's images via a video camera, creates the database, and realizes different recognition algorithms. The probability of correct target recognition varies from 0.6 for the IMF algorithm to 0.85 for the correlation algorithm.

The unimodal systems are characterized by a disadvantage - a vulnerability of the sensor to bad or noisy data as a result of the imperfect acquisition of captured features. This limitation can influence the results of the correct recognition.

\section{Model of the multimodal target recognition system}

The multimodal systems consist of a set of sensors and processing modules, every one of which realizes one algorithm for target recognition [20]. Multimodal TRS is more reliable than a unimodal system because many independent recognition modalities are used [21].

Let the functions $T_{k}(x, y), k=1 \div K$, describe the patterns generated by different sensors (Figure 3 ). For every function, $T_{k}(x, y)$ the respective recognition algorithm $A_{k}$ will be used which will permit the recognition of the target with a probability $\mathrm{p}_{\text {Ak. }}$.

In this case, the probability of correct target recognition in the multimodal system can be described as:

$$
P_{M M S}=1-\prod_{k=1}^{k}\left(1-p_{A k}\right)
$$

were $\Pi$ is the product function.

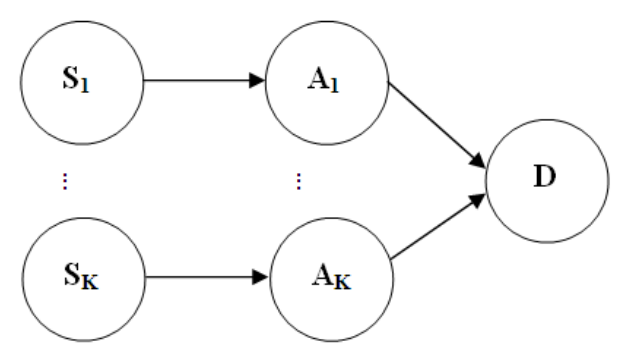

Figure 3. The graph presentation of the multimodal recognition system. 


\section{Multiple classification algorithm recognition systems}

To increase the reliability of TRS a new approach was proposed - to use a set of classification algorithms in the systems. This approach will permit the development of new kinds of systems - Multiple Classification Algorithms Unimodal and Multimodal Systems.

\subsection{Multiple classification algorithms unimodal system}

In the Multiple Classification Algorithms Unimodal System (MAUMS) one sensor is used and a set of the recognition algorithms is employed (Figure 4). Let $T(x, y)$ describe the input target, to which will be applied a set of the recognition algorithms $\left\{A_{q}\right\}, q=1 \div Q$. As a result will be obtained a set of matrixes $\left\{D_{A q}\right\}, q=1 \div Q$, each of which will be characterized by a probability of correct target recognition $\mathrm{p}_{\mathrm{Aq}}$.

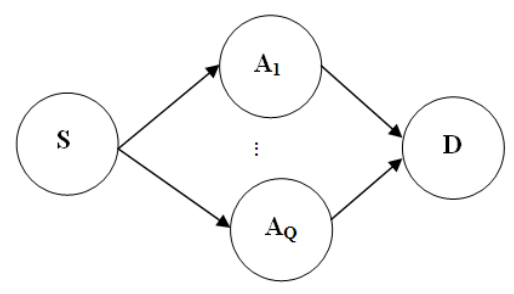

Figure 4. The graph presentation of the MAUMS system.

In this case, the correct recognition probability of the target $T(x, y)$ in the system can be evaluated as:

$$
P_{\text {MAUMS }}=\underset{q=1}{\stackrel{Q}{\prod}\left(1-p_{\text {Aq }}\right)}
$$

\subsection{Multiple classification algorithms multimodal system}

In the Multiple-Algorithm Multimodal System (MAMMS) a set of the sensors $\left\{\mathrm{S}_{i}\right\}, \mathrm{i}=1$ $L$ is used and the data from them are processed by different recognition algorithms (Figure 5 ). Let the functions $T_{l}(x, y), l=1 \div L$, describe the input targets. For every function $T_{l}(x, y)$ a set of the recognition algorithms $\left\{A_{\mathrm{l} z}\right\}, z=1 \div Z_{\text {l }}$ will be applied, which will permit the recognition of the target with a probability $\mathrm{p}_{\mathrm{Alz}}$. In this case, the probability of correct target recognition in the system can be described as:

$$
\begin{aligned}
& \text { L Zl } \\
& P_{\text {MAMMS }}=1-\Pi\left\{\Pi\left(1-p_{\text {Alz }}\right)\right\}
\end{aligned}
$$

\section{Analysis of the systems' target recognition probabilities}

The probability of the correct target recognition evaluation was made in the different systems based on the formulas (1), (3), (5), and (7). For evaluation there were selected the unimodal system (UMS); multimodal systems with one algorithm and 2 and 3 sensors (MMS$1 \mathrm{~A} 2 \mathrm{~S}$ and MMS-1A3S); multi-algorithm unimodal systems with 2 and 3 algorithms and one sensor (MAUMS-2A1S and MAUMS-3A1S), multi-algorithm multimodal systems with 2 algorithms and 2 sensors (MAMMS-2A2S), with 3 algorithms and 2 sensors (MAMMS-3A2S) and with 3 algorithms and 3 sensors (MAMMS-3A3S). For simplicity, it was supposed that the recognition probability $\mathrm{p}_{\mathrm{A}}$ of different algorithms is the same. The results of the calculations are presented in Table 1 and Figure 6, and show the following. The most effective are the 
multi-algorithm multimodal systems (MAMMS). The recognition effectiveness of the system MAMMS-3A3S is equal to 1.0 starting from the algorithm's identification probability $p_{A}=0.7$. The recognition effectiveness of the systems MMS-1A2S and MAUMS-2A1S, of the MMS-1A3S and MAUMS-3A1S, of the MAMMS-3A2S and MAMMS-2A3S is the same.

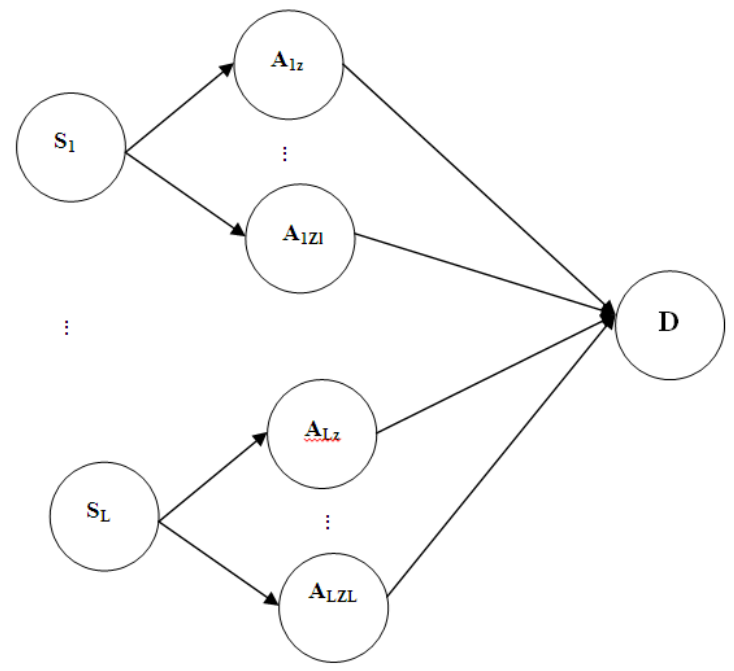

Figure 5. The graph presentation of the MAMMS system.

The maximal value of the algorithm's recognition probability $\mathrm{p}_{\mathrm{AM}}$ is proposed to determine for an established threshold level of the system's recognition probability P $_{\text {ST. The }}$ values of $\mathrm{p}_{\mathrm{AM}}$ will describe the requirements for the quality and, respectively, the costs of the recognition algorithms used in the systems. In the last row of Table 1 and Figure 7, the values $\mathrm{p}_{\mathrm{AM}}$ are presented for $\mathrm{P}_{\mathrm{ST}} \geqslant 0.99$. It is evident, that in the MAMMS systems the requirements for recognition algorithms are lowest $-\mathrm{p}_{\mathrm{AM}}=0.5-0.55$.

Table 1

Recognition probability of the systems, $P_{S}$

\begin{tabular}{|c|c|c|c|c|c|c|c|c|c|}
\hline$p_{A}$ & $\begin{array}{l}\text { UMS- } \\
\text { 1A1S }\end{array}$ & $\begin{array}{l}\text { MMS- } \\
1 A 2 S\end{array}$ & $\begin{array}{l}\text { MMS- } \\
1 A 3 S\end{array}$ & $\begin{array}{c}\text { MAUMS } \\
- \\
\text { 2A1S }\end{array}$ & $\begin{array}{c}\text { MAUMS } \\
- \\
\text { 3A1S } \\
\end{array}$ & $\begin{array}{c}\text { MAMMS } \\
- \\
2 A 2 S \\
\end{array}$ & $\begin{array}{c}\text { MAMMS } \\
- \\
3 A 2 S \\
\end{array}$ & $\begin{array}{c}\text { MAMMS } \\
- \\
2 A 3 S \\
\end{array}$ & $\begin{array}{c}\text { MAMMS } \\
-3 A 3 S\end{array}$ \\
\hline 0.5 & 0.5 & 0.75 & 0.875 & 0.75 & 0.875 & 0.9375 & 0.98438 & 0.98438 & 0.99805 \\
\hline 0.55 & 0.55 & 0.7975 & 0.90887 & 0.7975 & 0.90887 & 0.95899 & 0.9917 & 0.9917 & 0.99924 \\
\hline 0.6 & 0.6 & 0.84 & 0.936 & 0.84 & 0.936 & 0.9744 & 0.9959 & 0.9959 & 0.99974 \\
\hline 0.65 & 0.65 & 0.8775 & 0.95713 & 0.8775 & 0.95713 & 0.98499 & 0.99816 & 0.99816 & 0.99992 \\
\hline 0.7 & 0.7 & 0.91 & 0.973 & 0.91 & 0.973 & 0.9919 & 0.99927 & 0.99927 & 0.99998 \\
\hline 0.75 & 0.75 & 0.9375 & 0.98438 & 0.9375 & 0.98438 & 0.99609 & 0.99976 & 0.99976 & 1.00 \\
\hline 0.8 & 0.8 & 0.96 & 0.992 & 0.96 & 0.992 & 0.9984 & 0.99994 & 0.99994 & 1.00 \\
\hline 0.85 & 0.85 & 0.9775 & 0.99663 & 0.9775 & 0.99663 & 0.99949 & 0.99999 & 0.99999 & 1.00 \\
\hline 0.9 & 0.9 & 0.99 & 0.999 & 0.99 & 0.999 & 0.9999 & 1.00 & 1.00 & 1.00 \\
\hline 0.95 & 0.95 & 0.9975 & 0.99988 & 0.9975 & 0.99988 & 0.99999 & 1.00 & 1.00 & 1.00 \\
\hline$p_{A M}$ & - & 0.9 & 0.8 & 0.9 & 0.8 & 0.7 & 0.55 & 0.55 & 0.5 \\
\hline
\end{tabular}




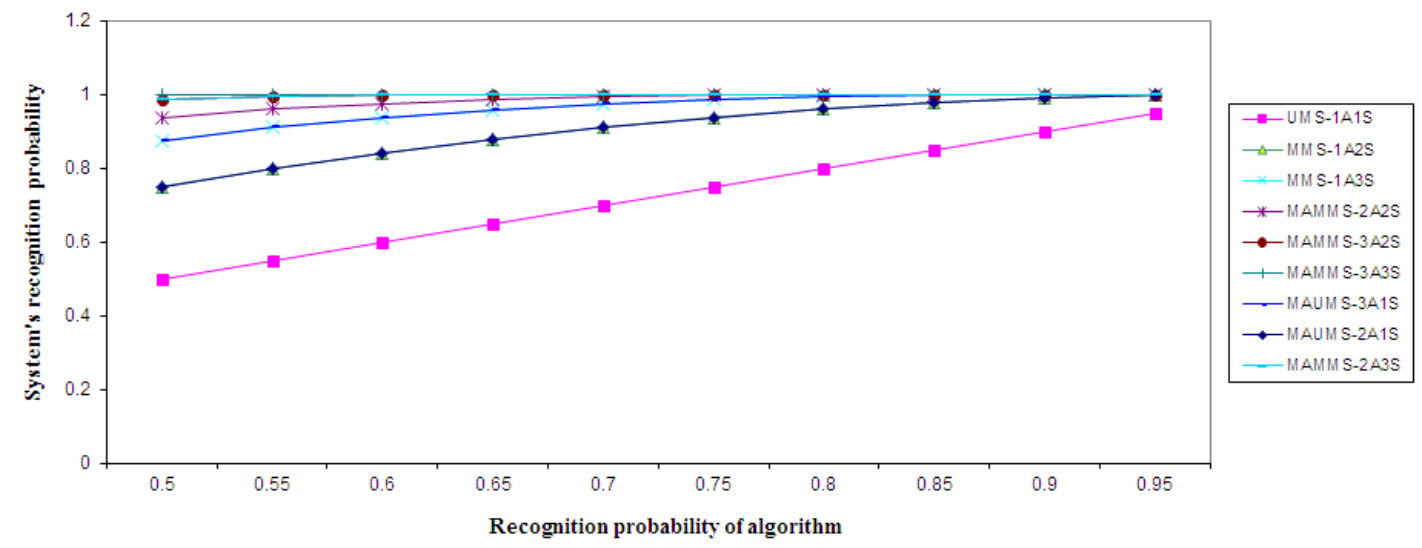

Figure 6. Recognition probability of systems.

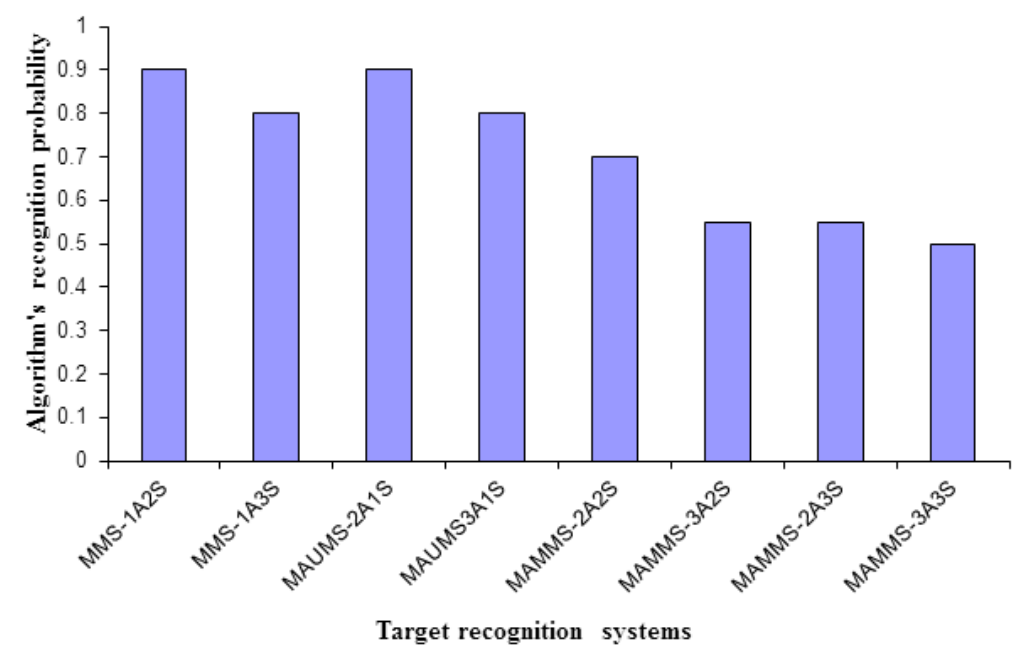

Figure 7. Algorithm's recognition probability $p_{A M}$ values at the system's recognition probability threshold level $\mathrm{P}_{\mathrm{ST}}=0.99$.

\section{Conclusions}

The general structure and main components of a target recognition system are presented. The characteristics such as availability, distinctiveness, robustness, and accessibility are described, which influence the reliability of a TRS.

The graph presentations and mathematical descriptions of a unimodal and multimodal TRS are given. The mathematical models for a probability of correct target recognition in these systems are presented. The unimodal systems are characterized by a disadvantage - a vulnerability of the sensor to bad or noisy data as a result of the imperfect acquisition of captured features.

To increase the reliability of TRS, a new approach was proposed - to use a set of classification algorithms in the systems. This approach permits the development of new kinds of systems - Multiple Classification Algorithms Unimodal and Multimodal Systems (MAUMS and MAMMS).

The graph presentations, mathematical descriptions of the MAUMS and MAMMS are described. The evaluation of the correct target recognition was made for different systems: the unimodal system (UMS); multimodal systems with one algorithm and 2 and 3 sensors (MMS-1A2S and MMS-1A3S); multi-algorithm unimodal systems with 2 and 3 algorithms and one sensor (MAUMS-2A1S and MAUMS-3A1S), multi-algorithm multimodal systems with 2 
algorithms and 2 sensors (MAMMS-2A2S), with 3 algorithms and 2 sensors (MAMMS-3A2S) and with 3 algorithms and 3 sensors (MAMMS-3A3S).

It was established that the most effective are the MAMMS systems. The recognition effectiveness of the system MAMMS-3A3S is equal to 1.0 starting from the algorithm's identification probability $p_{A}=0.7$. The recognition effectiveness of the systems MMS-1A2S and MAUMS-2A1S, of the MMS-1A3S and MAUMS-3A1S, of the MAMMS-3A2S and MAMMS$2 \mathrm{~A} 3 \mathrm{~S}$ is the same.

The conditions of systems' effectiveness were established. For $\mathrm{p}_{\mathrm{A}}<0.55$ the system MAUMS-2A1S is more effective than other systems. For $p_{A}<0.7$ the system MAUMS-3A1S is more effective than the system MMS-1A2S and the system MAMMS-2A2S is more effective than the system MMS-3S. The system MAMMS-3A2S is more effective in comparison with the system MAMMS-2A3S.

The modality of the algorithm's recognition probability $\mathrm{p}_{\mathrm{AM}}$ maximal value determination for an established threshold level of the system's recognition probability $P_{\text {ST }}$ was proposed. The values of $\mathrm{p}_{\mathrm{AM}}$ will describe the requirements for the quality and, respectively, the costs of the recognition algorithms. It was determined that in the MAMMS systems the requirements for recognition algorithms are lowest for $\mathrm{P}_{\mathrm{ST}} \geqslant 0.99$.

It was established that the proposed target recognition systems of the MAMMS kind ensure higher recognition probability in comparison with other systems, and they can use more simple and, respectively, cheaper recognition algorithms.

The proposed theory permits the system's design depending on a predetermined recognition probability. In the future, computer modeling and simulation of the proposed systems will be made and testing on the different kinds of targets.

\section{References}

1. Khan M., Yousaf A., Javed N. Automatic Target Detection in Satellite Images using Deep Learning. Journal of Space Technology, Vol. 7, No 1 (2017).

2. Hussain M., Bird J., Faria D. A Study on CNN Transfer Learning For Image Classification. 18th Annual UK Workshop on Computational Intelligence, Nottingham (2018).

3. DOAA, M., HASSANEIN, A., HASSANIEN, E. An Automatic Detection of Military Objects and Terrorism Classification System Based on Deep Transfer Learning. Proc. of Intern. Conf. on Artificial Intelligence and Computer Vision, p. 594 - 603, (2020).

4. Uribe-Hurtado A., Orozco-Alzate M. Acceleration of Dissimilarity-Based Classification Algorithms Using Multi-core Computation. Proc. Intern. Conf. on Practical Applications of Agents and Multi-Agent Systems, p. 231 - 233 (2017).

5. Ebbutt G., Thales R. New Intelligence, Surveillance, and Reconnaissance Concepts. Janes, 23 Sept. 2019. https://www.janes.com/defence-news/news-detail/thales-reveals-new-isr-concepts.

6. Jagruti J., Mehzabeen A. Object Recognition Using CNN. Intern. Journal of Advanced Research, Ideas, and Innovations in Technology. Vol. 4, Issue 2, p. 1987 - 1991 (2018).

7. Schachter B. Automatic Target Recognition. Proc. SPIE TT118, (2018).

8. Clemente, C., Pallotta, L., Gaglione, D. Automatic Target Recognition of Military Vehicles with Krawtchouk Moments. IEEE Trans. on Aerospace and electronic systems. Vol. 53, Issue 1, p. 493 - 500 (2017).

9. Gurbuz A. C., Robiulhossain M., Bedri C. A Cognitive Radar Target Detection and Tracking With Multifunctional Reconfigurable Antennas. IEEE Aerospace and electronic systems magazine. Vol. 35, Nr. 6 p. 64 - 76 (2020).

10. Qu Y., Wang T., Zhu Z. Vision-aided Laser Doppler Vibrometry For Remote Automatic Voice Detection. IEEE/ASME Trans. Mechatron. p. 1110 - 1119 (2011). DOI: 10.1109/TMECH.2010.2077678.

11. Cho H., Seo Y.W., Kumar B.V., Rajkumar R. A Multi-Sensor Fusion System For Moving Object Detection And Tracking In Urban Driving Environments; Proceedings of the 2014 IEEE International Conference on Robotics and Automation (ICRA); Hong Kong, China. 31 May-7 June 2014; pp. 1836 - 1843. 
12. Makantasis K., Protopapadakis E., Doulamis A., Matsatsinis N. Semi-supervised Vision-Based Maritime Surveillance System Using Fused Visual Attention Maps. Multimed. Tools Appl. Vol. 75, p. 1- 28. (2016). DOI: 10.1007/s11042-015-2512-x.

13. Yufu Q., Guirong Z., Zhaofan Z. Active Multimodal Sensor System for Target Recognition and Tracking. Sensors. Vol. 17, Issue 7. (2017). Published online 2017 Jun 28. DOI: 10.3390/s17071518. https://www.ncbi.nlm.nih.gov/pmc/articles/PMC5539591/

14. Qu Y., Wang T., Zhu Z. Vision-aided Laser Doppler Vibrometry for Remote Automatic Voice Detection. IEEE/ASME Trans. Mechatron. Vol. 16, p.1110 - 1119. (2011). DOI: 10.1109/TMECH.2010.2077678.

15. Gao Z., Dai J., Xie C. Dim and Small Target Detection Based On Feature Mapping Neural Networks. Journal of Visual Communication and Image Representation. Vol. 62. p. 206 - 216. (2019).

16. Woollard M., Bannon A., Ritchie M., Griffiths H. Synthetic Aperture Radar Automatic Target Classification Processing Concept. Electronics Letters. Vol. 55, Nr. 24, p. 1301 - 1303. (2019).

17. Correll B., Beard J., Swanson C. Cost as Array Waveforms for Closely Spaced Target Detection. IEEE Trans. on Aerospace and electronic systems. Vol. 56, Nr. 2, p. 1045 - 1076. (2020).

18.Geng Z., Deng H., Himed B. Ground Moving Target Detection Using Beam-Doppler Image Feature Recognition. IEEE Trans. on Aerospace and electronic systems. Vol.54, Nr. 5, p. 2329 - 2341. (2018).

19. Perju V., Casasent D., Ciubotaru O. The Systems for Persons' Identification and Verification Based On Face Correlation Recognition. Proc. SPIE 6245 (2006).

20. Shuang Y., Xiaoran S., Feng Z. Automatic Target Recognition for Low-Resolution SAR Images Based on SuperResolution Network. Proc. of the 6th Asia-Pacific Conference on Synthetic Aperture Radar Conf. November (2019). DOI: 10.1109/APSAR46974.2019.9048251.

21. Silva H., Rad P., Beebe N., et all. Cooperative Unmanned Aerial Vehicles With a Privacy-Preserving Deep Vision For Real-Time Object Identification And Tracking. Journal of parallel and distributed computing. Vol. 131, p. 147 - 160. (2019). 